\title{
Improving MEG source localizations: An automated method for complete artifact removal based on independent component analysis
}

\author{
D. Mantini, ${ }^{*}$ R. Franciotti, G.L. Romani, and V. Pizzella \\ Institute of Advanced Biomedical Technologies, “G. D'Annunzio University” Foundation, Chieti, Italy \\ Department of Clinical Sciences and Bio-imaging, “G. D’Annunzio” University, Chieti, Italy
}

Received 4 September 2007; revised 9 October 2007; accepted 13 November 2007

Available online 3 December 2007

\begin{abstract}
The major limitation for the acquisition of high-quality magnetoencephalography (MEG) recordings is the presence of disturbances of physiological and technical origins: eye movements, cardiac signals, muscular contractions, and environmental noise are serious problems for MEG signal analysis. In the last years, multi-channel MEG systems have undergone rapid technological developments in terms of noise reduction, and many processing methods have been proposed for artifact rejection. Independent component analysis (ICA) has already shown to be an effective and generally applicable technique for concurrently removing artifacts and noise from the MEG recordings. However, no standardized automated system based on ICA has become available so far, because of the intrinsic difficulty in the reliable categorization of the source signals obtained with this technique. In this work, approximate entropy (ApEn), a measure of data regularity, is successfully used for the classification of the signals produced by ICA, allowing for an automated artifact rejection. The proposed method has been tested using MEG data sets collected during somatosensory, auditory and visual stimulation. It was demonstrated to be effective in attenuating both biological artifacts and environmental noise, in order to reconstruct clear signals that can be used for improving brain source localizations.
\end{abstract}

(c) 2007 Elsevier Inc. All rights reserved.

Keywords: Approximate entropy; Artifact removal; Blind source separation; Independent component analysis; Magnetoencephalography; Source localization

\section{Introduction}

Magnetoencephalography (MEG), first measured by David Cohen in 1968, is a noninvasive technique that permits the study

\footnotetext{
* Corresponding author. ITAB - Istituto Tecnologie Avanzate Biomediche, Fondazione "Università G. D'Annunzio", Università degli Studi di Chieti “G. D'Annunzio", Via dei Vestini 33, 66013 Chieti, Italy. Fax: +39 08713556930 .

E-mail address: d.mantini@unich.it (D. Mantini).

Available online on ScienceDirect (www.sciencedirect.com).
}

of the magnetic brain activity at the millisecond level, providing an important insight into the human brain function (Cohen, 1968, 1972; Del Gratta et al., 2001). MEG signals are associated with electrical currents produced by large populations of neurons that depolarize almost synchronously (Hari et al., 1997) or simultaneously respond to a single stimulus (Williamson et al., 1991). In order to measure these particularly weak signals, whose maximum amplitude is at level of hundreds of femtoTesla, superconducting quantum interference device (SQUID) sensors, invented and developed in 1964 at the Ford Scientific Laboratory (Dearborn, MI, USA), are used for their high sensitivity to magnetic fields (Jaklevic et al., 1964, Pizzella et al., 2001). Nonetheless, the most important difficulty for the acquisition of high-quality MEG recordings is the presence of noise from physiological and technical origins that could corrupt the measurements (Ziehe et al., 2000). The most common processing method for increasing signal-to-noise ratio (SNR) is the averaging of the responses from multiple stimuli. This procedure reduces both ambient field noise and brain activity that are uncorrelated with the stimulus, although SNR does not increase indefinitely because the evoked field might be attenuated in case of a large number of stimuli. Additionally, averaging epochs is not possible for some functional studies, as for example those related to brain rhythms (Liljestrom et al., 2005). With the aim of increasing SNR in single trials (Vrba and Robinson, 2001), several other signal processing methods for attenuating noise and artifacts have been developed, as for example signal space projection (Nolte and Curio, 1999; Uusitalo and Ilmoniemi, 1997) and signal space separation (Taulu et al., 2004). Among all them, one of the most promising approaches is based on independent component analysis (ICA), which was applied first to EEG signals by Makeig in 1996 (Makeig et al., 1996), and subsequently to MEG signals by Vigário (Vigário et al., 1998).

ICA is a signal processing technique that is able to separate independent components (ICs) produced by distinct sources from linearly mixed signals (Comon, 1994; Hyvärinen and Oja, 2000; James and Hesse, 2005). The fundamental limitation of this approach is unquestionably the necessity of classifying the source signals after their separation; this is a nontrivial task, because in 
general there is no a priori information on how many components will be related to disturbances and to cerebral activity, and it is impossible to predict the order with which all components will be sorted. Although methods using several combined criteria for the detection of artifactual components has been proposed for assisting investigators during the categorization of components (Barbati et al., 2004; Rong and Contreras-Vidal, 2006), the use of ICA for MEG processing still relies on the manual identification of the recovered signals (Moran et al., 2004). On the other hand, the automation of an ICA-based method for the reconstruction of artifact-free signals would be a great step toward a wider clinical application of MEG.

In this paper, we describe a completely automated method for the retrieval of clear brain signals from multi-channel MEG recordings. ICA was used to separate independent source signals; these were then categorized with a dedicated algorithm using approximate entropy (ApEn), which is a measure of data regularity. ApEn quantifies the predictability of subsequent amplitude values of a physiological signal based on the knowledge of the previous amplitude values (Pincus, 1991; Pincus and Goldberger, 1994). To the best of our knowledge, this is the first time that ApEn is applied for the automated classification of ICs extracted from MEG recordings. Our findings showed that the ICA approach, integrated with ApEn for the IC classification, demonstrated to be an appropriate method for MEG data processing. In particular, the SNR of the reconstructed brain signals is generally higher than that provided by standard processing methods, and also the consistency of MEG source localizations seems to increase.

\section{Materials and methods}

\section{MEG data acquisition}

We used 12 MEG data sets from healthy right-handed male subjects (age 20-35 years) for the optimization and the validation of the proposed method. They were acquired for 6 previously conducted event-related field (ERF) studies (Del Gratta et al., 2002; Stavrinou et al., 2007; Della Penna et al., 2007; Perfetti et al., 2007), whose details are summarized in Table 1.

With regard to data acquisition in all studies, the magnetic field was recorded by using a multi-channel MEG system (ATB, Pescara, Italy), consisting of 153 dc-SQUID-integrated magnetometers with a sensitivity of approximately $8 \mathrm{fT} \mathrm{Hz}^{-1 / 2}$ at $1 \mathrm{~Hz}$ and $5 \mathrm{fT} \mathrm{Hz}^{-1 / 2}$ above $10 \mathrm{~Hz}$ (Della Penna et al., 2000). The MEG system was located inside a magnetically shielded room characterized by a shielding factor of $70 \mathrm{~dB}$ at $1 \mathrm{~Hz}$. MEG hardware recording parameters were $1025 \mathrm{~Hz}$ sampling rate and 0.16-250 Hz band-pass filtering. To determine the position of the subject head with respect to the sensors, the magnetic field generated by four coils placed on the scalp was recorded before and after each measurement session. A coordinate system was defined by digitizing anatomical landmarks on the subject head by the 3D digitizer 3Space Fastrak (Polhemus, Colchester, VT, USA).

Magnetic resonance imaging was used to co-register MEG functional data with brain anatomical images, and express the source coordinates into the Talairach space. A set of highresolution images (voxel size $1 \mathrm{~mm}^{3}$, MPRAGE sequence) were obtained by a Magnetom Vision scanner (Siemens, München, Germany) operating at $1.5 \mathrm{~T}$.
Table 1

Details of the ERF study protocols

\begin{tabular}{cllll}
\hline \multicolumn{1}{c}{$\begin{array}{l}\text { Stimulation } \\
\text { type }\end{array}$} & $\begin{array}{l}\text { Stimulation } \\
\text { characteristics }\end{array}$ & $\begin{array}{l}\text { Acquisition } \\
\text { time (min) }\end{array}$ & $\begin{array}{l}\text { Interstimulus } \\
\text { time (s) }\end{array}$ \\
\hline $\begin{array}{c}\text { Subjects } \\
1-2\end{array}$ & Somatosensory & $\begin{array}{l}\text { Right median nerve } \\
\text { electric stimulation } \\
\text { (motor threshold) }\end{array}$ & 3.3 \\
$\begin{array}{c}\text { Subjects } \\
3-4\end{array}$ & Somatosensory & $\begin{array}{l}\text { Right tibial nerve } \\
\text { electric stimulation } \\
\text { (motor threshold) }\end{array}$ & 6 & 3 \\
Subjects & Somatosensory & $\begin{array}{l}\text { Right 2nd finger } \\
\text { electric stimulation } \\
\text { (sensitive threshold) }\end{array}$ & 8 & 1.4 \\
Subjects & Somatosensory & $\begin{array}{l}\text { Right-hand } \\
\text { stimulation with } \\
7-8\end{array}$ & $\begin{array}{l}\text { pin-electrode } \\
\text { (painful threshold) } \\
\text { Monotonic } \\
\text { listening (syllable } \\
\text { from right side) }\end{array}$ & 3 \\
Subjects & Auditory & $\begin{array}{l}\text { Bar pattern reversal } \\
\text { (top right quadrant) }\end{array}$ & 2.5 & 2.3 \\
$9-10$ & Subjects & Visual & & 0.5 \\
$11-12$ & & & \\
\hline
\end{tabular}

The characteristics of the stimulations are provided, along with the corresponding acquisition times and interstimulus times.

\section{Separation of independent components}

MEG recordings were pre-processed with a notch filter at $50 \mathrm{~Hz}$ for power line disturbance rejection, and with a Chebyshev II-type filter (band-pass $1.0-80.0 \mathrm{~Hz}$ ) for an initial environmental noise reduction.

Subsequently, ICA was used to separate the source signals mixed in the MEG data sets. ICA theory assumes that a set of observations of $q$ random variables $g(t)=\left[g_{1}(t), \ldots, g_{q}(t)\right]^{T}$ is produced by an instantaneous linear mixture of $p$ independent source signal components $s(t)=\left[s_{1}(t), \ldots, s_{p}(t)\right]^{T}$. Therefore, the generative ICA model, describing how the observed data are generated by a process of mixing the source signals, can be expressed by

$g(t)=\mathbf{A} s(t)+n(t)$

where $\mathbf{A}$ is a constant $[q \times p]$ matrix called mixing matrix, and $n(t)$ is a vector of white noise, of the same dimension as $g(t)$. The minimal required a priori information for this model is the independence of the source signals. Furthermore, a solution for the ICA problem is possible if two additional conditions are met: the number of underlying signals is at most equal to the number of observations $(p \leq q)$, and the mixing matrix is full column-rank ( $r$ $(\mathbf{A})=p$ ). In this case, the independent components (ICs) can be retrieved by determining a $[p \times q]$ matrix $\mathbf{W}$, named unmixing matrix, for which the $p$-dimensional vector

$\hat{s}(t)=\mathbf{W} g(t)$

is the best estimate of the vector $s(t)$.

The ICA decomposition of the MEG recordings into source signals was performed by means of the FastICA algorithm (Hyvärinen, 1999), freely available at www.cis.hut.fi/projects/ica/ fastica, because it proved to achieve fast convergence also in case of a large number of observations, and to be less sensitive to white noise than other ICA algorithms (Hyvärinen and Oja, 2000). The 
ICA decomposition is achieved by FastICA through the nonGaussianity maximization of the estimated source signals. The non-Gaussianity is expressed in terms of negentropy $J$, defined as the difference between the entropy $H$ of a Gaussian random variable $s_{\text {gauss }}$ (having the same mean and variance of the observed random variable $s$ ) and the entropy of $s$ :

$J(\hat{s})=H\left(\hat{s}_{\text {gauss }}\right)-h(\hat{s})$

FastICA was run using a deflation scheme (Hyvärinen and Oja, 1997) for sequentially obtaining the columns of the unmixing matrix W, and hence retrieving the ICs according to (2). An algorithm that estimates a single IC and subtracts it from the MEG recordings with proper weights was iteratively run. The procedure was automatically stopped when FastICA was not able to converge, i.e. when the residual signal for all MEG channels was white noise.

When the estimate of the unmixing matrix $\mathbf{W}$ was completed, the mixing matrix A was computed by means of a pseudo-inversion

$\mathbf{A}=\left(\mathbf{W}^{T} \mathbf{W}\right)^{-1} \mathbf{W}^{T}$

The $i$-th column of the matrix A, containing the amplitudes of the $i$-th component in the recordings, was then used to retrieve the power $P_{i}$, expressed by

$P_{i}=\sum_{j=1}^{q} a_{j i}^{2}$

Accordingly, the ICs were sorted with decreasing power level (James and Hesse, 2005).

\section{Automated component classification}

The automated IC classification was based on approximate entropy (ApEn), a chaos-theory feature useful for the characterization of complex systems. ApEn of a time series measures the logarithmic likelihood that runs of patterns of length $m$ that are close to each other will remain close in the next incremental comparisons, $m+1$. A larger likelihood of remaining close (high regularity) produces smaller ApEn values, and, conversely, low regularity produces higher ApEn values (Pincus, 1991).

Given a sequence $S_{N}$ composed by $N$ consecutive samples of the signal $s_{i}(t)$, ApEn can be calculated, given that two input parameters have been chosen: the previously introduced parameter $m$ specifies the pattern length, and the parameter $r$ defines the criterion of similarity. The pattern is a subsequence of $m$ consecutive values, beginning from the instant $i$ within $S_{N}$, denoted by the vector $p_{m}(i)$. Two patterns $p_{m}(i)$ and $p_{m}(j)$ are similar if the difference between any pair of corresponding measurements in the patterns is less than $r$, i.e. if

$\left|\hat{s}_{i+k}-\hat{s}_{j+t}\right|<r \quad$ for $\quad 1 \leq t \leq m$

Considering the set $P_{m}$ of all patterns of length $m$, i.e. $\left\{p_{m}(1)\right.$, $\left.p_{m}(2), \ldots, p_{m}(N-m)\right\}$, within $S_{N}$, the quantity $C_{i}^{m}(r)$ can be defined as

$C_{i}^{m}(r)=\frac{n_{i}^{m}(r)}{N-m}$

where $n_{i}^{m}(r)$ is the number of patterns in $P_{m}$ that are similar to $p_{m}$ (i). The entropy of the empirical distribution arising on the observed subset of $P_{m}$ can be indicated by the parameter $\Phi^{m}$, defined as

$\Phi^{m}(r)=\frac{1}{N-m} \sum_{i=1}^{N-m} \ln C_{i}^{m}(r)$

ApEn is then quantified as

$\operatorname{ApEn}(m, r, N)=\Phi^{m}-\Phi^{m+1}=\frac{1}{N-m}\left[\sum_{i=1}^{N-m} \ln \left(\frac{C_{i}^{m}(r)}{C_{i}^{m+1}(r)}\right)\right]$

The ICs were classified into three groups related to the MEG sources, i.e. cerebral activations, non-cerebral biological signals, environmental disturbances. A tailored procedure was used, in order to obtain a single reference value of ApEn for each IC. Each component was filtered in the band $1.0-40 \mathrm{~Hz}$, resampled at $100 \mathrm{~Hz}$ and divided into time windows of $10.240 \mathrm{~s}$ (1024 samples); for each time window, an ApEn value was then computed using $m=2, r=0.2 * \mathrm{SD}, N=1024$, in order to obtain a reliable signal characterization (Pincus, 1991; Pincus and Goldberger, 1994); the reference ApEn value for the single IC was finally obtained as the median among all those retrieved (from 15 to 48, depending on the data set).

ApEn, being an entropy feature, is expected to be large for environmental magnetic noise and small for non-cerebral biological signals (e.g. cardiac signal, ocular movements), whereas the brain signals are supposed to be characterized by intermediate values. Consequently, optimal thresholds for separating the distributions and categorizing the source signals were required. The corresponding cutoff values were determined in order to achieve the maximum partition between the ApEn values of brain signals with respect to those of biological artifacts and environmental noise.

\section{Artifact-free MEG signal reconstruction}

The output of the ApEn based procedure was used to divide the source signals $\mathrm{s}_{\mathrm{i}}(\mathrm{t})$ separated by FastICA into two categories: brain signals and disturbances (both biological and nonbiological). The classification was used to create a $[p \times p]$ diagonal matrix $\mathbf{D}$; each element $d_{i i}$ was set equal to 0 if the $i$-th component was a brain signal, and it was set equal to 1 otherwise. The vector of clear MEG recordings $g_{\mathrm{c}}(t)$ could be then obtained by

$g_{\mathrm{c}}(t)=g(t)-\mathbf{A D} \hat{s}(t)$

In this manner, the artifacts were subtracted from the MEG recordings with appropriate weights for each channel,

Fig. 1. MEG data from subject 1. (Top panel) Illustrative example of $10 \mathrm{~s}$ of raw traces, corresponding to 10 recording channels widely distributed over the whole-head helmet. The ECG signal is also shown. (Bottom panel) Sample segments of the 15 ICs separated with ICA from the same data set. The amplitudes are arbitrary because the components are normalized. For each of them, the manual classification is provided aside $(\mathrm{B}=$ biological non-cerebral, $\mathrm{S}=$ brain $\mathrm{signal}$, $\mathrm{N}=$ environmental noise). 


\section{Raw MEG recordings}

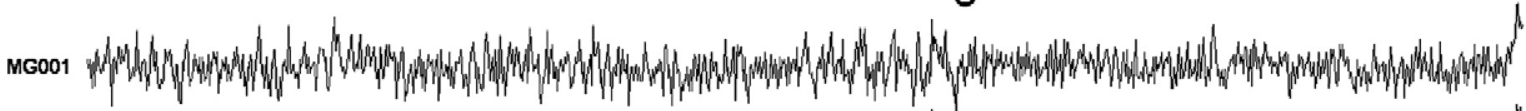

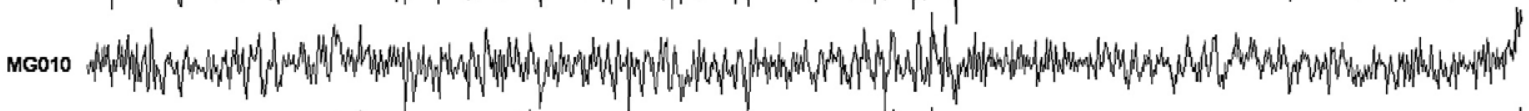

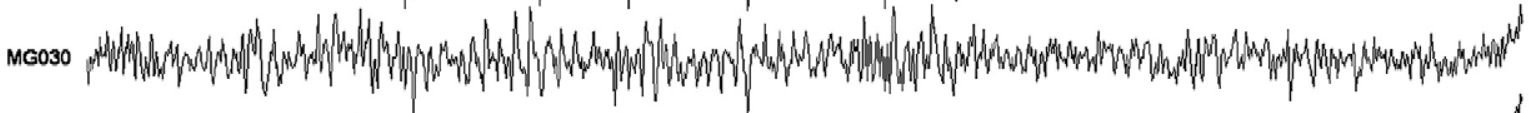

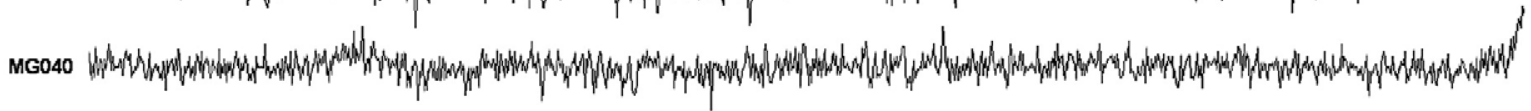

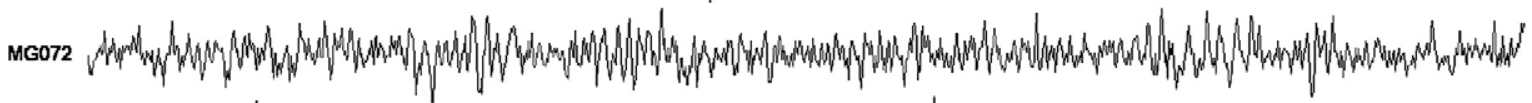

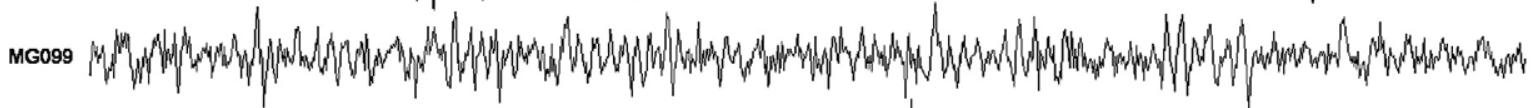

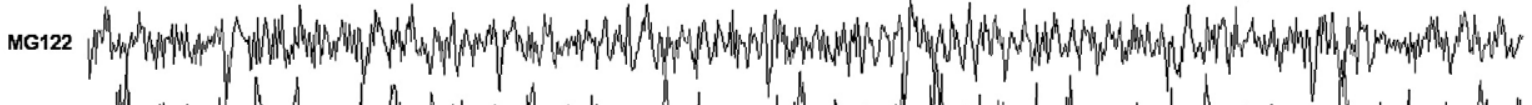
ma139.

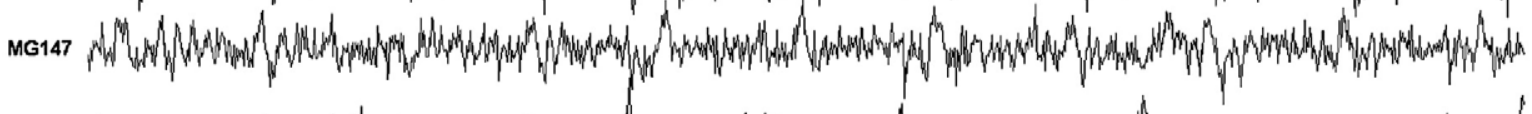

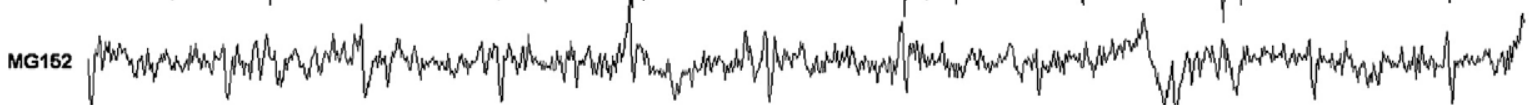
I

\section{Independent components}

Ico

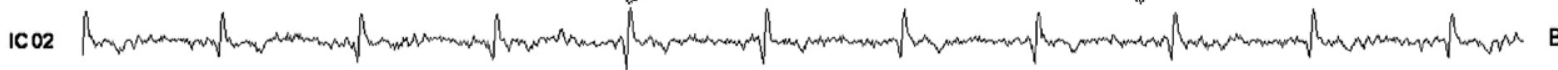

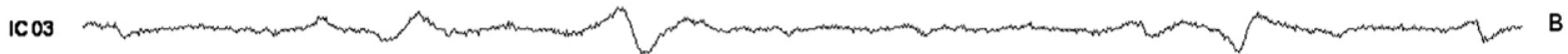

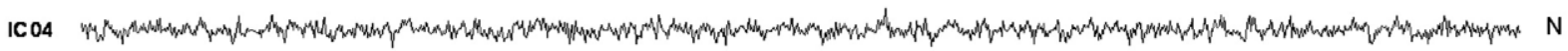
IC 05 strom

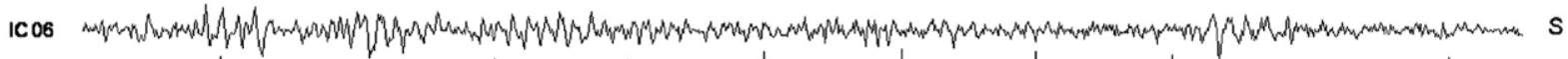

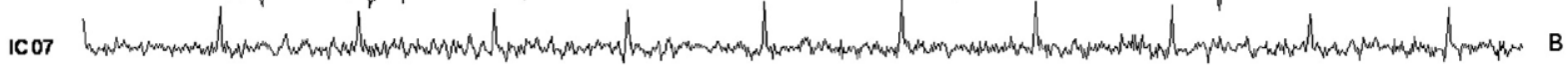

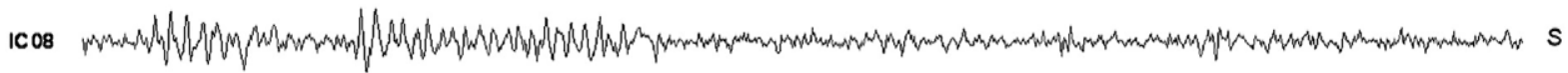

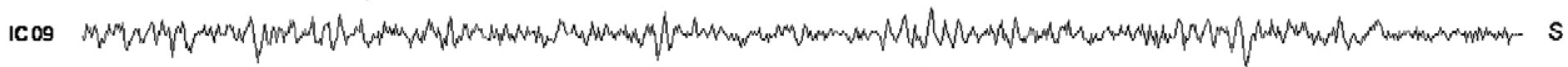

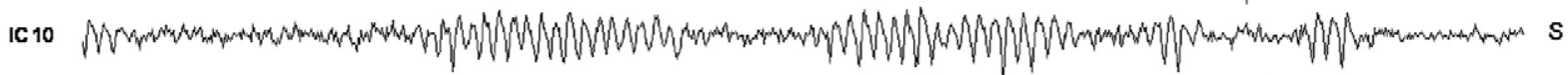
IC11

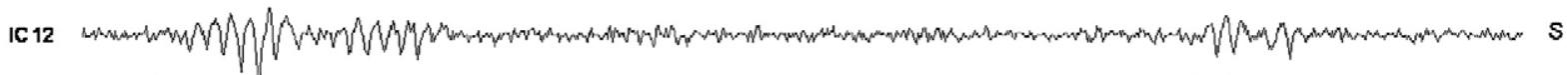

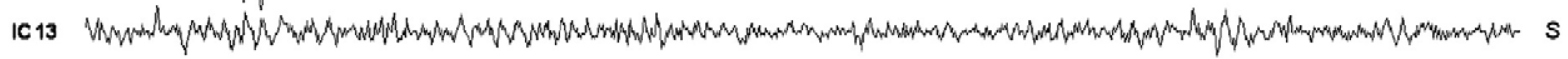

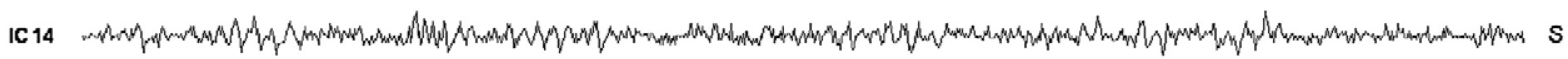

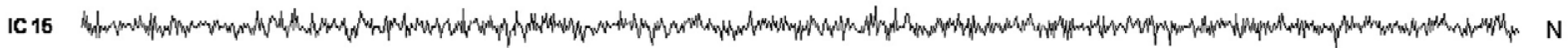

\begin{tabular}{|c|c|c|c|c|c|c|c|c|c|c|}
\hline 0 & 1 & 2 & 3 & 4 & $\begin{array}{c}5 \\
\text { conc }\end{array}$ & 6 & 7 & 8 & 9 & 10 \\
\hline
\end{tabular}


and the direct reconstruction of artifact-free MEG signals was accomplished.

\section{Validation of the automated method}

The validation of the automated method for the complete artifact removal included the analysis of the data sets with regard to two aspects: the categorization of the source signals, and the reconstruction of high-quality MEG signals.

After the ICA decomposition for all data sets, a manual classification of the resulting ICs was performed by three independent investigators. The general criteria used for the categorization were based on the observation of the IC timecourse and power spectrum, and of the associated MEG flux map (Scherg and Berg, 1991): ocular components were identified by means of all these three information patterns; cardiac and muscular components by means of time-course and flux map; cerebral and noise components by means of power spectrum and flux map. When the investigator was not able to make an uncomplicated decision, the specific IC was considered as an unknown component. For the creation of the group manual classification, each IC was ascribed to a specific class whether it ranked at least two out of three selections by the investigators.

After the creation of the manual classification for all 12 MEG data sets, we divided them into the two subsets characterized by even and odd number code, namely the learning and test groups. Each group contained 6 data sets from the same ERF studies (see Table 1 for more details). The learning group was used to set the thresholds required for the automated method; the test group was then used to assess the efficacy of the classification system.

Using the learning group, we associated the ApEn values obtained for all ICs with the reference manual classification, and therefore we compared the frequency distributions of ApEn values found for the three signal categories. A kernel smoothing function allowed the retrieval of probability density estimates for each category (Zhou et al., 2002). Using them, receiver operating characteristic (ROC) curves were calculated for assessing the separation between biological artifacts and brain signals, and between brain signals and environmental noise. The thresholds for the optimal separation of the categories were directly obtained from the two ROC curves, finding for each of them the ApEn value for which specificity and sensitivity were equal (Zhou et al., 2002).

After the selection of the ApEn thresholds, the number of errors in the ICs classification was computed for each signal category using the data sets belonging to the test group. A numerical parameter was used to quantify the overall performance of the classification system, namely the independent component detection rate (ICDR), defined as the ratio between the total number of correctly detected ICs and the total number of analyzed ICs.

Also the reconstruction of high-quality MEG signals was assessed on the test group, by comparing the signals restored by means of ICA with those obtained using a standard processing (SP) method. The latter consisted of band-pass filtering between 1.0 and $80 \mathrm{~Hz}$ (as for ICA processing), rejection of epochs containing ocular and muscular artifacts, cancellation of cardiac signal by means of an adaptive algorithm based on orthogonal projections (Samonas et al., 1997). The performances of the ICA-based method were tested in terms of: (1) cardiac artifact attenuation; (2) ocular and muscular artifact removal; (3) overall noise reduction; (4) ECD localization effectiveness.
The efficacy in the attenuation of the cardiac artifact was evaluated from the MEG data, respectively, before and after the artifact rejection. The QRS complexes in the ECG channel were used as trigger points, in order to calculate average cardiac signals from the MEG recordings. The average peak-to-peak cardiac artifact amplitude of the signals obtained before ICA processing was computed; then, the average peak-to-peak cardiac artifact amplitude was extracted using the traces after ICA and SP, respectively.

The ability of ICA to remove ocular and muscular artifacts was tested setting an amplitude threshold to the MEG recordings: all epochs with amplitude larger than $10 \mathrm{pT}$ were assumed to contain these kinds of artifacts and were considered not to be useful for further analysis. Therefore, the total number of acquired epochs was compared with the number of epochs available for analysis after standard and ICA processing, respectively.

The noise amplitude, measured from averaged signals, was calculated as the root mean square (RMS) of the signal values in the prestimulus interval. The same procedure was used to calculate the noise amplitude in the average signals obtained after SP and ICA, as well as the noise amplitude in the average signals obtained from raw MEG traces.

The localization performances were assessed with respect to the first dipolar pattern of the evoked response. The time intervals used for the localizations were identified using the average signals processed with SP. The signal-to-noise ratio (SNR) was estimated as the ratio between the maximum signal amplitude in the selected time interval and average noise level calculated from the prestimulus interval. The equivalent current dipole (ECD) model in the homogeneous sphere was used for MEG source localizations (Phillips et al., 1997; Scherg and Berg, 1991) by means of the BESA software package (MEGIS Software GmbH, Gräfelfing, Germany). The ECD position $R$ in the Talairach space was determined for the ERFs processed with ICA and SP, respectively. The stability of ECD localizations was tested dividing the total number of epochs in 10 equivalent parts, and calculating the average signals for each of them. The ECD positions $R_{k}$ with $k=1$, ..., 10 were estimated, and the distances $d_{k}$ between $R_{k}$ and $R$ were calculated. The localization variability was finally measured as the average value of the vector $d=\left[d_{1}, \ldots, d_{10}\right]$ : the smaller this parameter is, the more consistent the reference ECD position would be.

\section{Results}

All recordings contained cardiac and ocular artifacts, as well as environmental noise; in some cases also muscular artifacts and stimulation artifacts were found. An illustrative example of some MEG traces, referring to subject 1 (median nerve galvanic stimulation), is shown in Fig. 1. For each data set, the ICA method allowed the separation of disturbances of various origins, as well as brain sources with different spatio-temporal characteristics. In general, 7 to 15 ICs were retrieved, depending on the specific data set. The complete set of the ICs separated from the data set of subject 1 is shown in the Fig. 1, along with the outcome of the manual classification. In this case, two ICs corresponding to cardiac artifact, one to ocular artifact, and two to environmental noise were detected. Fig. 2 shows examples of the plots that had been used for the manual categorization of brain signals and disturbances, referring to 4 different ICs in time and frequency domain and the related MEG field distribution. 


\section{IC 01}

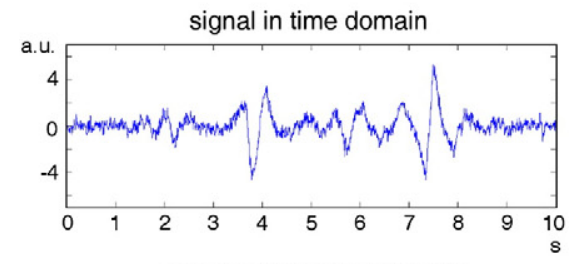

signal in frequency domain

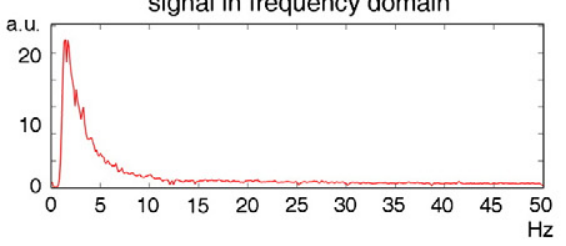

MEG flux map
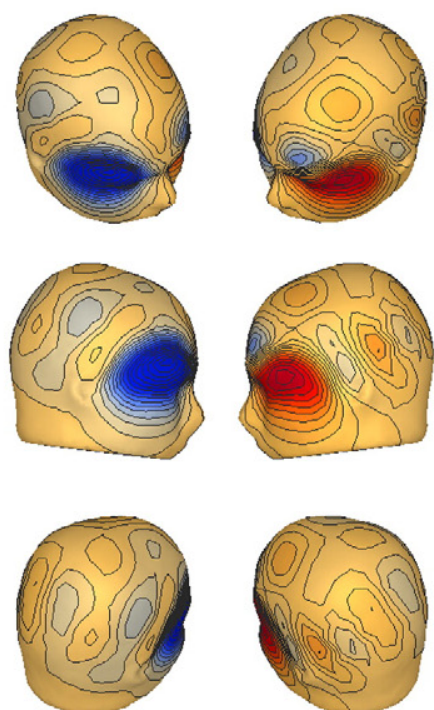

a.u.
IC 02

signal in time domain

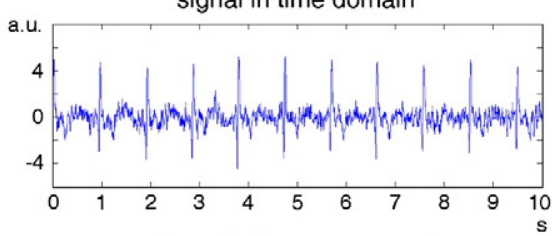

signal in frequency domain

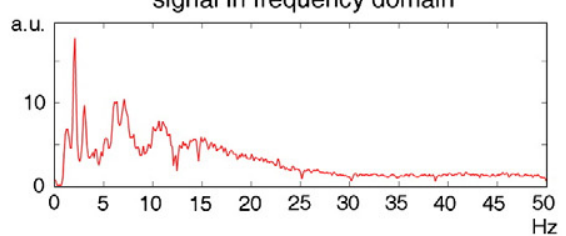

MEG flux map
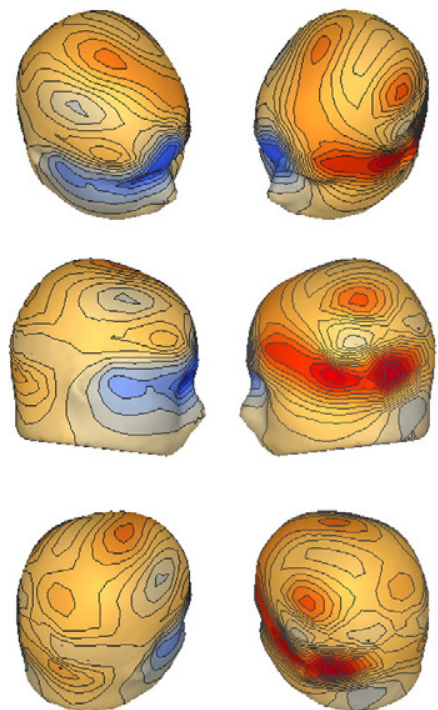

a.u

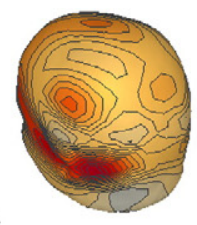

m
IC 04

signal in time domain

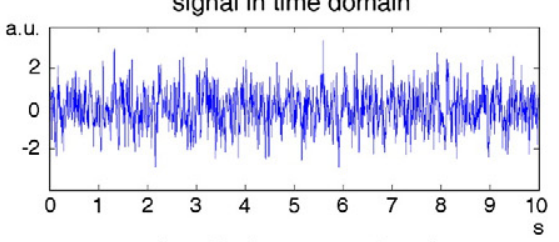

signal in frequency domain

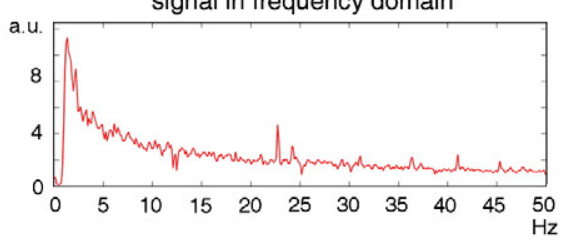

MEG flux map
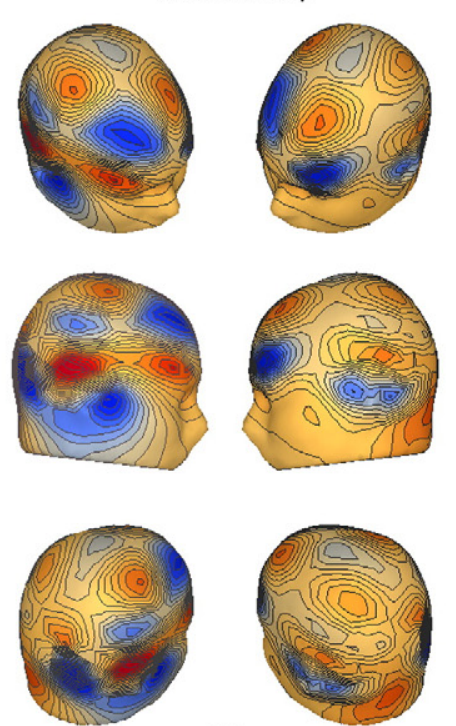

a.u.
IC 09

signal in time domain

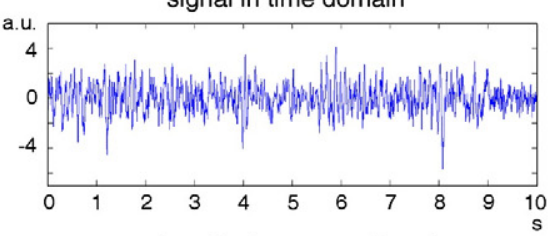

signal in frequency domain

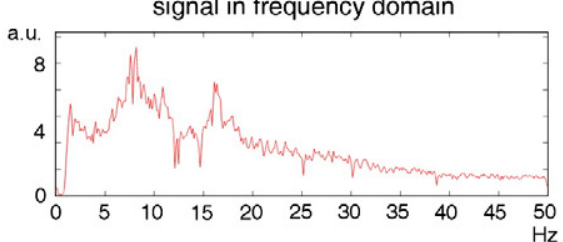

MEG flux map
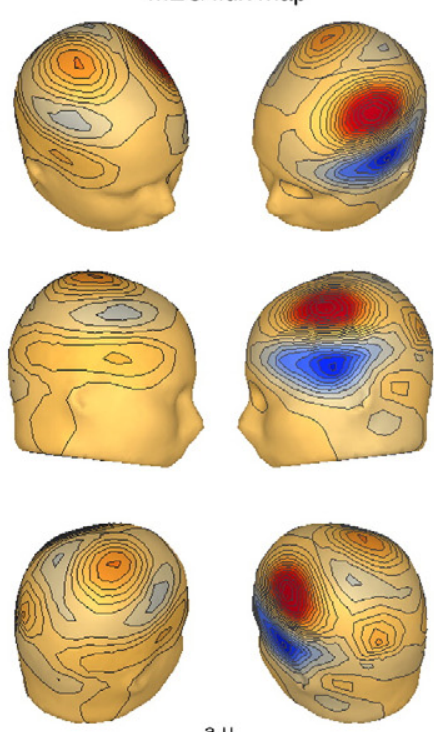

a.u.

Fig. 2. Example of the information used for the ICs classification: signals in time domain, spectra and MEG flux maps. IC_01, IC_02 and IC_04 are classified as ocular artifact, cardiac artifact and environmental noise, respectively. IC_09 is a brain signal, whose MEG flux map shows a dipolar distribution that is consistent with an electrophysiological activation in the cSI region. 
IC_01 is an ocular component, showing a characteristic $1 / f$ trend in the power spectrum and a magnetic field with larger intensity over the frontal regions; IC_02 is a cardiac component, for which the time-course encloses the typical heart QRS complexes and the power spectrum is largely between 1 and $30 \mathrm{~Hz}$; IC_04 is an environmental component, showing narrow peaks at different frequencies and an $1 / \mathrm{f}$ trend in the power spectrum, and an irregular MEG field distribution; IC_09 is a cerebral component with a power spectrum in the alpha and beta bands, and a dipolar flux map that may be consistent with a source in the cSI region.

Using the $6 \mathrm{MEG}$ data sets belonging to the learning group, the ApEn thresholds for an optimal separation of the three IC categories were obtained. Among the 89 separated components, 19 were categorized by the investigators as biological non-cerebral, 45 as cerebral, and 35 as noise components. The histograms of the ApEn values corresponding to the IC classes are presented in Fig. 3. The lowest ApEn values are related to cardiac, muscular and ocular signals and the highest to background noise; the ApEn values of brain signals are generally in-between. The large values of the area under the ROC curves, which are illustrated in Fig. 4, confirmed the significant separation of the three IC classes. Furthermore, these plots permitted the selection of adequate thresholds for an optimal classification of the ICs. The cutoff values that were obtained for the separation of cerebral signals from biological artifacts and environmental noise were 1.32 and 1.57 , respectively. The outcomes of the automated classification method that was based on these thresholds are extensively presented in Table 2 for the MEG data sets belonging to the test group: in summary, we achieved an overall ICDR equal to $91.4 \%$, compared to the manual classification.

After the identification of the disturbances, the latter are subtracted from the MEG recordings with appropriate weights for each channel. The signals referring to subject 1 after ICA processing are shown in Fig. 5; they do not show any noticeable residual of the subtracted disturbances. The same signals after SP, which is based on the attenuation of the cardiac signal by means of orthogonal projections and the rejection of epochs containing ocular and muscular artifacts, are also illustrated in Fig. 5 for comparison. For all analyzed data sets, the ICA method seemed to provide a superior signal quality, due to a more effective artifact removal than SP. This finding is consistent with the results of the analytical comparison performed between the two techniques, which are summarized in Table 3: ICA resulted in a better cardiac artifact rejection with respect to SP, the removal of ocular artifacts allowed for an overall increase from $94.5 \%$ to $99.5 \%$ of epochs available for analysis, and the noise level was reduced of about $30 \%$.

Two examples of ERFs, referring to subjects 1 and 11, and obtained from data processed with SP and ICA methods, respectively, are shown in Fig. 6. For the first one, characterized by a satisfactory data quality, very small differences can be found in terms of waveforms and flux maps. With regard to the second one, the residual contribution of noise and artifacts is strongly reduced in the signals processed with ICA, which in particular seems to provide a reliable reconstruction of the magnetic field distribution. Moreover, when using the signals processed with ICA, the localizations obtained using the ECD model, illustrated in Fig. 7 and analytically presented in Table 4, are always consistent with the expected brain activations for the particular stimulation and wave latency. Conversely, the signals obtained with SP have
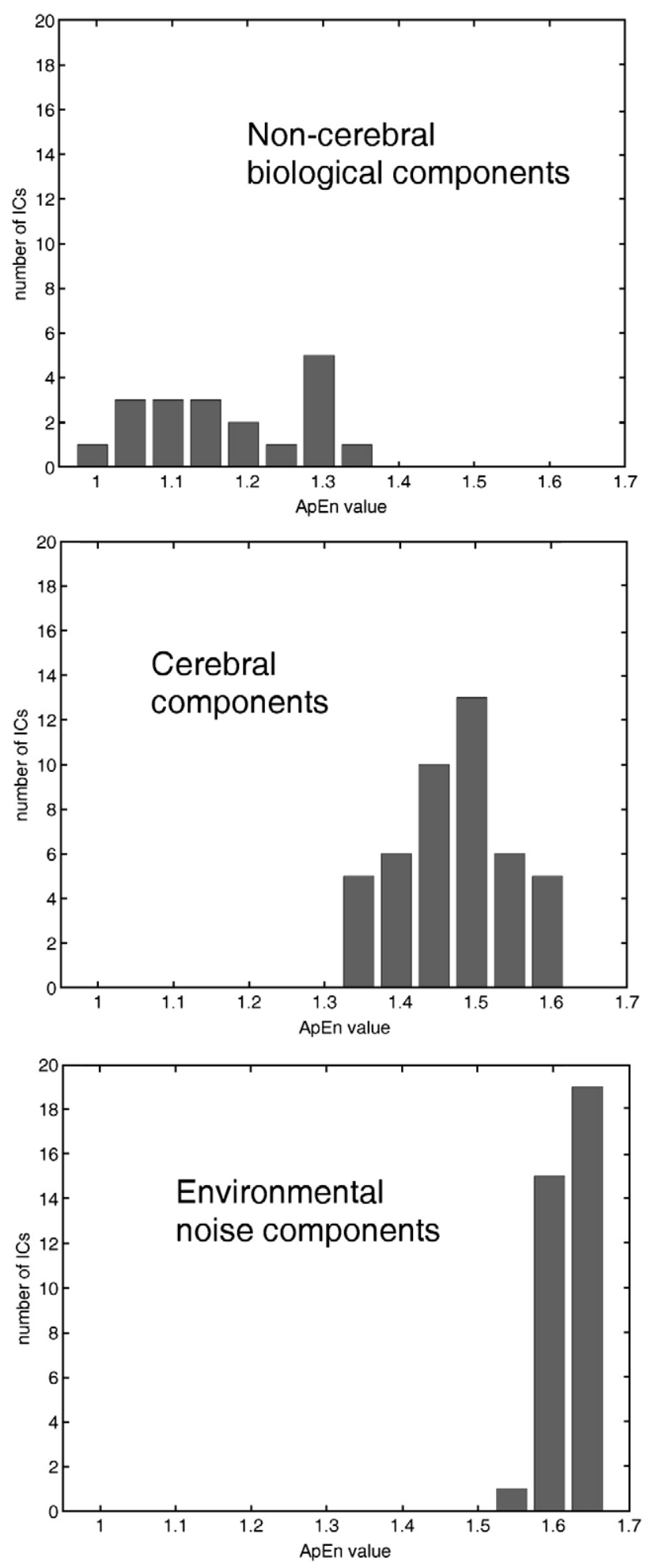

Fig. 3. Histograms of ApEn values for the three ICs categories: biological non-cerebral components, brain components, environmental noise components. The ApEn values were calculated for the ICs belonging to the learning group (MEG data sets with odd number code).

demonstrated to be less reliable for localization purposes, being characterized by generally larger values of the localization variability index, shown in Table 4. 

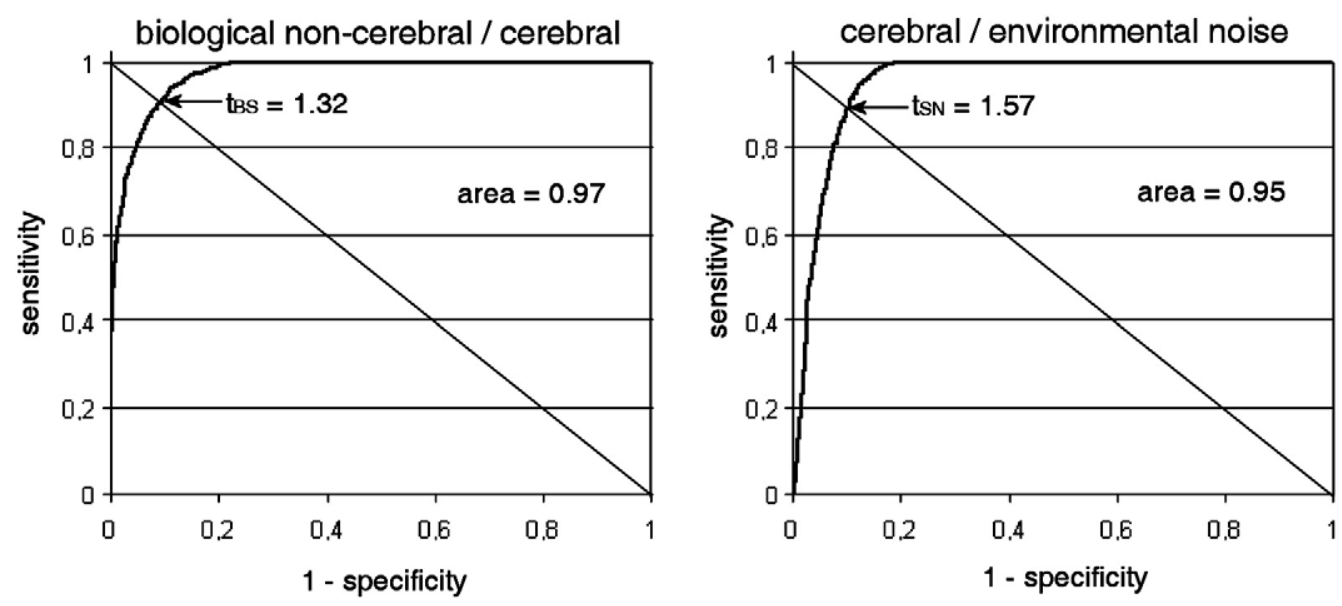

Fig. 4. Receiver operating characteristic curves. (Left panel) Separation between biological artifacts and cerebral signals. (Right panel) Separation between cerebral signals and environmental noise. The optimal cutoff ApEn value and the area under the ROC curve are also provided.

The whole ICA-based automated method for artifact removal, implemented in MATLAB and run on a PC with Pentium IV processor at $2.5 \mathrm{GHz}$ and $1.5 \mathrm{~GB}$ RAM, required a total computation time ranging between 20 and $50 \mathrm{~min}$, depending on the specific data set.

\section{Discussion}

\section{ICA decomposition}

ICA has shown in several fields its ability to recover the independent activity originating from distinct sources, without relying on a priori information about their dynamics and/or their spatial structure (Hyvärinen and Oja, 2000). In particular, ICA was demonstrated to be valuable in neuroimaging research for the extraction of source signals (Moran et al., 2004; Barbati et al., 2006), as well as for the attenuation of biological and nonbiological artifacts (Barbati et al., 2004; Rong and ContrerasVidal, 2006). In this study, we applied FastICA algorithm to data collected with our MEG system during somatosensory, auditory and visual stimulation.

Although our method for the ICA decomposition is comparable with other ICA-based methods in the MEG literature, it is specifically different with regard to the selection of the number of ICs. Previous works suggested the use of a full ICA decomposition with MEG data, with the number of ICs equal to that of recordings (Rong and Contreras-Vidal, 2006). However, if the number of sources that are linearly mixed in the recordings is overestimated, this approach might provide an unsatisfactory IC separation and might also produce a number of spurious components with low power. On the other hand, we used an iterative method for estimating the optimal number of ICs, retaining as much data variance as possible. We obtained in this manner a number of sources much lower than the number of the recording channels, as also found in several MEG studies (Fuchs et al., 2004; Vrba and Robinson, 2001; Wagner et al., 2004). However, it is also possible that, due to the strong spatial low-pass properties of the forward mapping, the brain sources essentially occupy a restricted subspace in channel space.

An intriguing aspect of ICA processing is that this method allows not only the partition between the disturbances and the brain-related signals, but also permits the separation of brain signals with different spatio-temporal patterns. Therefore, its use might be valuable not only for the study of the evoked responses but also for the investigation of the ongoing brain activity, for which no event-related averaging could be used for signal-to-noise ratio enhancement. It is worth noting, however, that ICA can present some limitations: the decomposition may occasionally be unsatisfactory, due for example to a high level of white noise in the recordings or to subject's movements during the acquisition. This problem in the signal separation phase could also affect the performances of the IC classification, in either manual or

Table 2

Performances of the automatic IC classification system for single data sets, compared to the manual selection

\begin{tabular}{|c|c|c|c|c|c|c|c|c|}
\hline & & Subject 1 & Subject 3 & Subject 5 & Subject 7 & Subject 9 & Subject 11 & All subjects \\
\hline \multirow{2}{*}{ Biological non-cerebral ICs } & Correct ICs & 3 & 4 & 4 & 3 & 3 & 1 & 18 \\
\hline & Missed ICs & 1 & 0 & 0 & 0 & 0 & 0 & 1 \\
\hline \multirow[t]{2}{*}{ Cerebral ICs } & Correct ICs & 8 & 6 & 3 & 2 & 7 & 3 & 29 \\
\hline & Missed ICs & 0 & 0 & 0 & 0 & 1 & 2 & 3 \\
\hline \multirow[t]{2}{*}{ Environmental noise ICs } & Correct ICs & 3 & 1 & 4 & 2 & 3 & 4 & 17 \\
\hline & Missed ICs & 0 & 1 & 0 & 0 & 1 & 0 & 2 \\
\hline \multirow{2}{*}{ All separated ICs } & Correct ICs & 14 & 11 & 11 & 7 & 14 & 8 & 65 \\
\hline & Missed ICs & 1 & 1 & 0 & 0 & 1 & 2 & 5 \\
\hline
\end{tabular}

The analysis was performed on the data sets belonging to the test group (odd number code). For each signal category, the number of manually detected components and the number of false negatives provided by the classification system are shown. No results for the group of unknown components are reported, as no ICs were labeled as unknown, according to the reference manual classification. 


\section{ICA artifact removal}

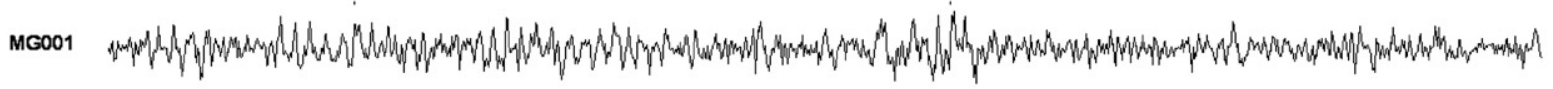

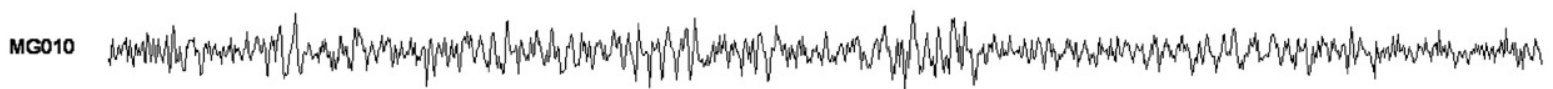

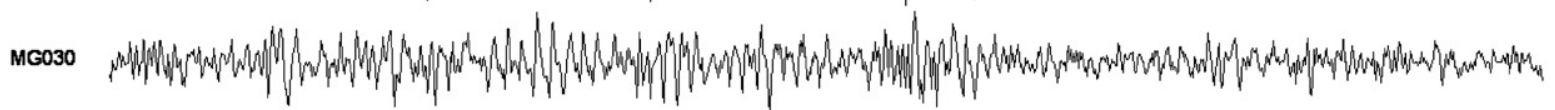

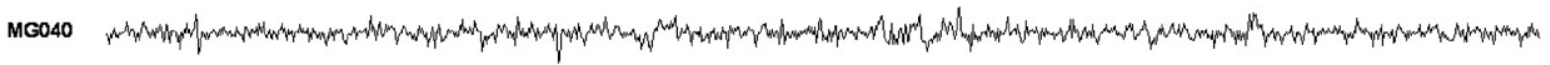

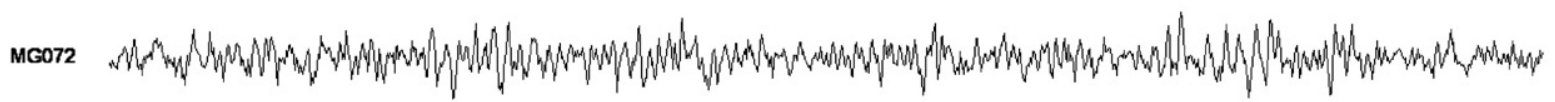

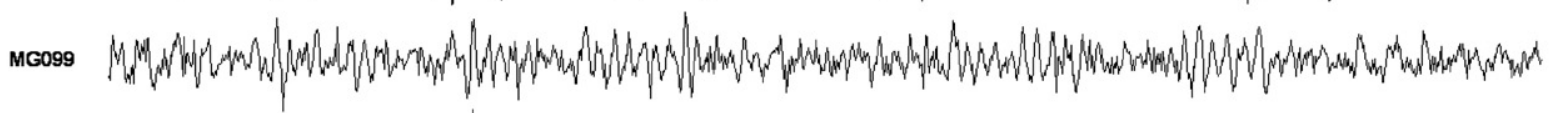

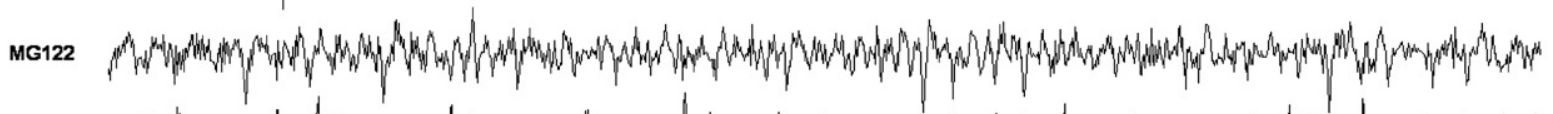

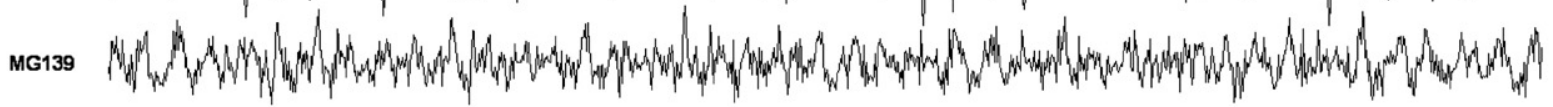

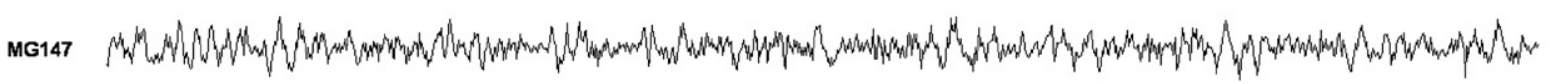

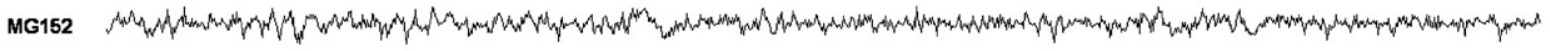

\section{SP artifact removal}

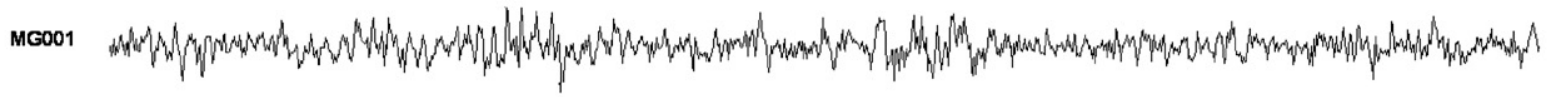

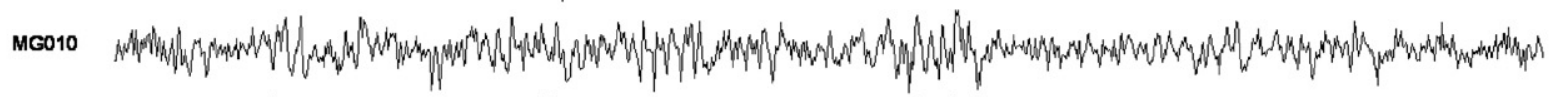

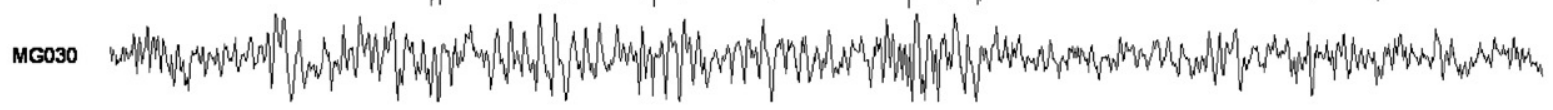
MG040

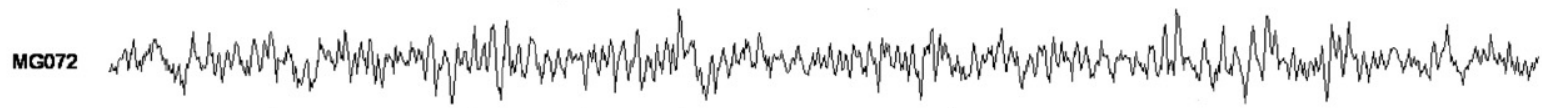

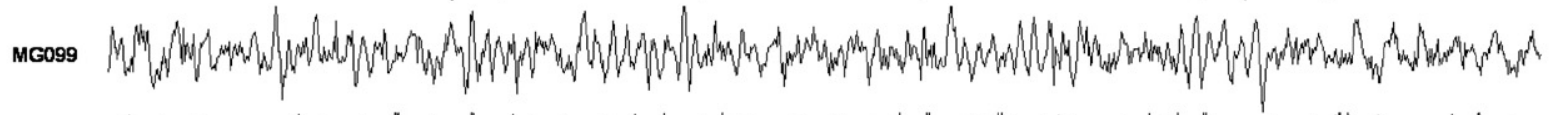

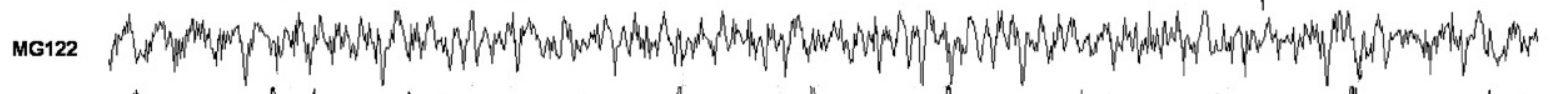

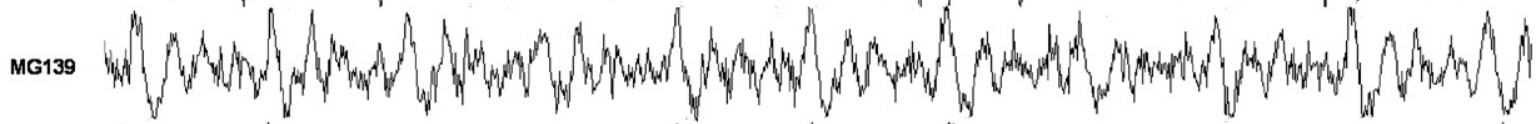

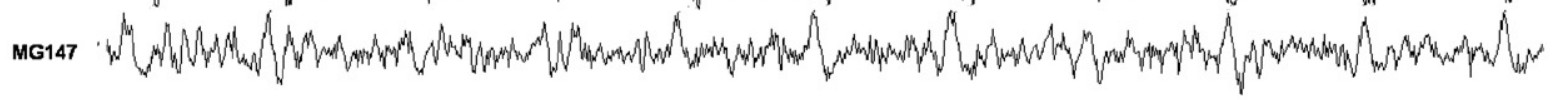

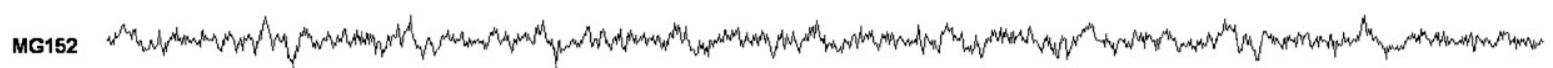

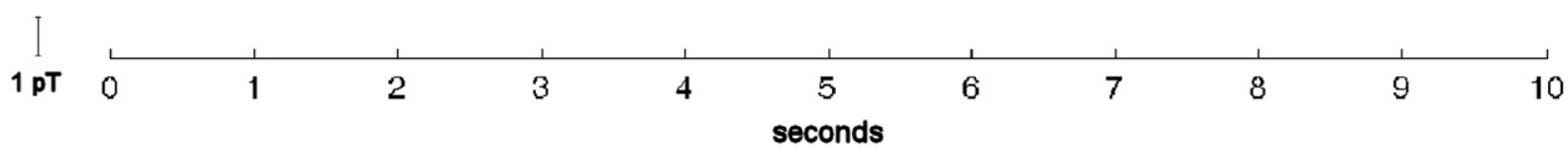

Fig. 5. Artifact-free MEG traces corresponding to the raw signals presented in Fig. 1. (Top panel) Signals reconstructed by means of ICA; (Bottom panel) Signals reconstructed by means of SP. 
Table 3

Performances of the artifact removal and quality of the reconstructed signals

\begin{tabular}{|c|c|c|c|c|c|c|c|}
\hline & & Subject 1 & Subject 3 & Subject 5 & Subject 7 & Subject 9 & Subject 11 \\
\hline \multirow[t]{3}{*}{ Cardiac artifact attenuation $^{\mathrm{a}}$} & Cardiac amplitude in raw traces $(\mathrm{pT})$ & $0.6 \pm 0.2$ & $3.4 \pm 3.1$ & $2.5 \pm 2.4$ & $0.7 \pm 0.5$ & $3.0 \pm 3.0$ & $3.3 \pm 3.3$ \\
\hline & Cardiac amplitude in traces after SP (pT) & $0.4 \pm 0.1$ & $1.0 \pm 0.7$ & $0.7 \pm 0.7$ & $0.3 \pm 0.2$ & $1.1 \pm 0.9$ & $0.5 \pm 0.5$ \\
\hline & Cardiac amplitude in traces after ICA (pT) & $0.1 \pm 0.1$ & $0.3 \pm 0.2$ & $0.2 \pm 0.2$ & $0.3 \pm 0.1$ & $0.4 \pm 0.3$ & $0.2 \pm 0.1$ \\
\hline \multirow{3}{*}{$\begin{array}{l}\text { Ocular and muscular artifact } \\
\text { attenuation }\end{array}$} & Total epochs in raw recordings & 92 & 113 & 326 & 95 & 72 & 263 \\
\hline & Available epochs after SP & 92 & 96 & 302 & 95 & 65 & 258 \\
\hline & Available epochs after ICA & 92 & 112 & 323 & 95 & 72 & 262 \\
\hline \multirow[t]{3}{*}{ Overall noise reduction $^{\mathrm{a}}$} & Noise amplitude before processing (fT) & $22 \pm 8$ & $35 \pm 23$ & $58 \pm 45$ & $42 \pm 26$ & $98 \pm 85$ & $45 \pm 38$ \\
\hline & Residual noise amplitude after SP (fT) & $21 \pm 7$ & $30 \pm 19$ & $55 \pm 43$ & $39 \pm 25$ & $80 \pm 67$ & $43 \pm 36$ \\
\hline & Residual noise amplitude after ICA (fT) & $18 \pm 7$ & $21 \pm 12$ & $26 \pm 17$ & $30 \pm 11$ & $73 \pm 61$ & $27 \pm 14$ \\
\hline
\end{tabular}

For each subject, the effectiveness in cardiac artifact attenuation can be appreciated from the cardiac amplitude of the traces processed with SP and ICA with respect to that of the raw MEG signals. The outcome of the ocular and muscular artifact removal is assessed comparing the numbers of epochs available for analysis after SP and ICA with the total number of acquired epochs. Noise amplitude in the prestimulus baseline is also shown for the average signals before and after artifact rejection. Amplitudes correspond to the mean value and to the standard deviation calculated using the values obtained from all the recording channels.

${ }^{\text {a }}$ Figures are expressed as mean value \pm standard deviation.

automated manner, as well as the reconstruction of artifact-free recordings.

\section{IC categorization}

A reliable automated IC classification system, able to isolate the artifactual signals, is particularly valuable with the ICA approach, because a high number of ICs are generally produced, and the manual procedure is in general very time consuming. Moreover, the use of an automated method would be appropriate, because it would standardize the whole ICA processing, eliminating inter-operator disagreements, eventually induced by the manual IC classification.

In our study, the proposed ApEn-based system has permitted a consistent categorization of the ICs. Noise components have shown high ApEn values, being ambient disturbances rather unpredictable. Conversely, biological non-cerebral components have provided low ApEn values, because muscular, cardiac and ocular artifacts are characterized by almost regular and predictable signal patterns. However, the ApEn values obtained for the three signal groups partially overlap in the distribution tails. This result can be ascribed to the presence, among the ICs, of biological artifacts contaminated by residual noise, and of cerebral signals characterized by very irregular intrinsic activity. As a consequence, the two cutoff thresholds were chosen for minimizing the detection errors, but it was impossible to achieve a perfect IC classification. However, we should mention that an imperfect classification does not necessarily prevent the reconstruction of consistent MEG traces, because the errors related to low-power ICs have a minimum influence on the reconstructed signals.

In addition, it is worth noting that the proposed detection system, which was directly developed for and tested on real MEG data sets, performed significantly better than other systems proposed in the literature. In particular, the ICDR of our system was much larger than $79 \%$, reported for another ICA-based system using statistical properties and spectral characteristics (Barbati et al., 2004).

\section{Artifact rejection}

The proposed ICA method, integrated with an IC classification tool, allows to detect and simultaneously remove cardiac, muscular, ocular artifacts, and background noise from the MEG recordings. ICA performs considerably better than the SP method for the cancellation of the cardiac signal. SP requires the use of a reference ECG channel, and it relies on the iterative subtraction of the averaged artifact. For this reason, it is affected by the variability in cardiac wave morphology and duration (Samonas et al., 1997); conversely, ICA is able to directly isolate the cardiac artifact components on the basis of their statistical independence from other source signals.

With regard to the muscular and ocular artifacts, the proposed method represents an enhancement with respect to the classical segment-rejection approach. ICA allowed to preserve almost all the recorded epoch, saving functional information from data loss and recovering the functional brain activities masked by artifactual signals. This is an important advantage, especially when limited MEG data are available. As a first consequence of the increase in the number of available epochs, the SNR of the averaged signals improves with respect to rejection-based methods. On the other hand, the lower values of overall noise in the ERFs can also be ascribed to a partial cancellation of artifacts and environmental noise from the recordings.

\section{ECD localization}

The comparative analysis of noise level in ERFs after SP and ICA methods demonstrates that the latter, being superior in the elimination of artifacts, is consequently able to provide more dependable signals. This is an important requirement, in particular for event-related studies that necessitate the identification of the correct timing of particularly weak waves. Moreover, it should be emphasized that ICA is able to reduce noise of about $30 \%$ and to recover useful MEG signals, also for some data sets that, conversely, would not be included into group studies because of their poor SNR. Moreover, the similarity of ECD localizations when the signal quality is satisfactory for both the two processing methods suggests that no systematic bias is induced by the ICA-based artifact rejection; nonetheless, the smaller values of localization variability obtained with ICA demonstrate the increased consistency of the source localizations that can be obtained using these processed signals.

\section{Conclusion}

MEG is currently considered a reliable method for the noninvasive monitoring of the electromagnetic brain activity by analyzing the magnetic fields associated with it (Del Gratta et al., 2001). This noninvasive imaging technique permits to obtain signals 


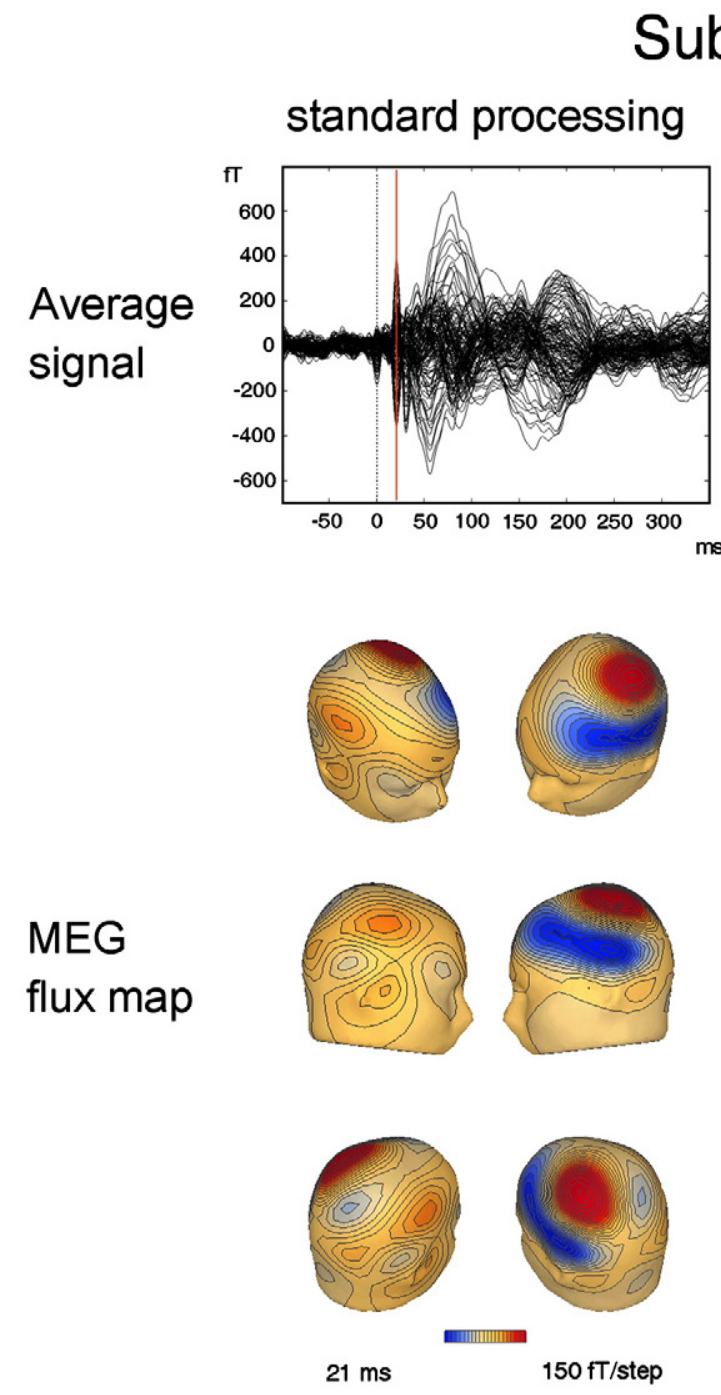

\section{ject 1}
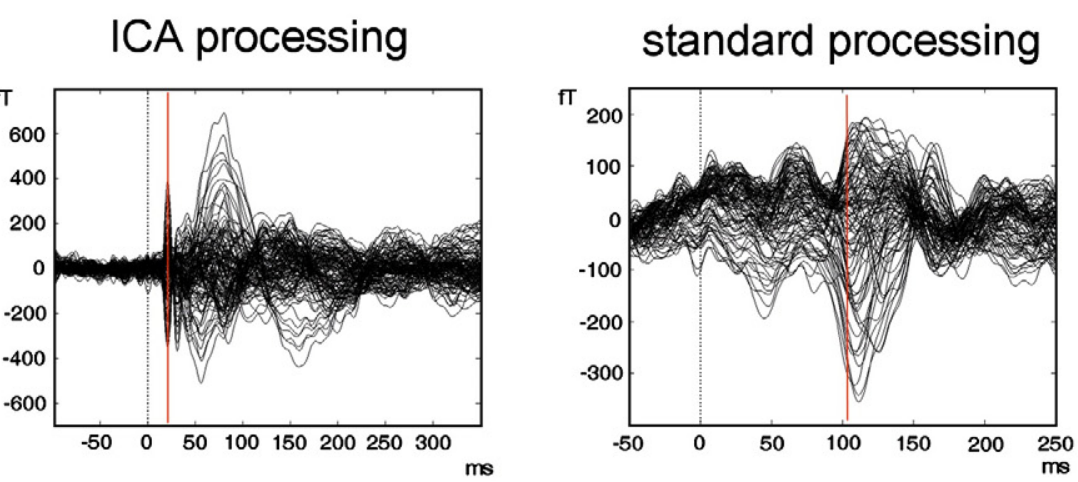

Subject 11
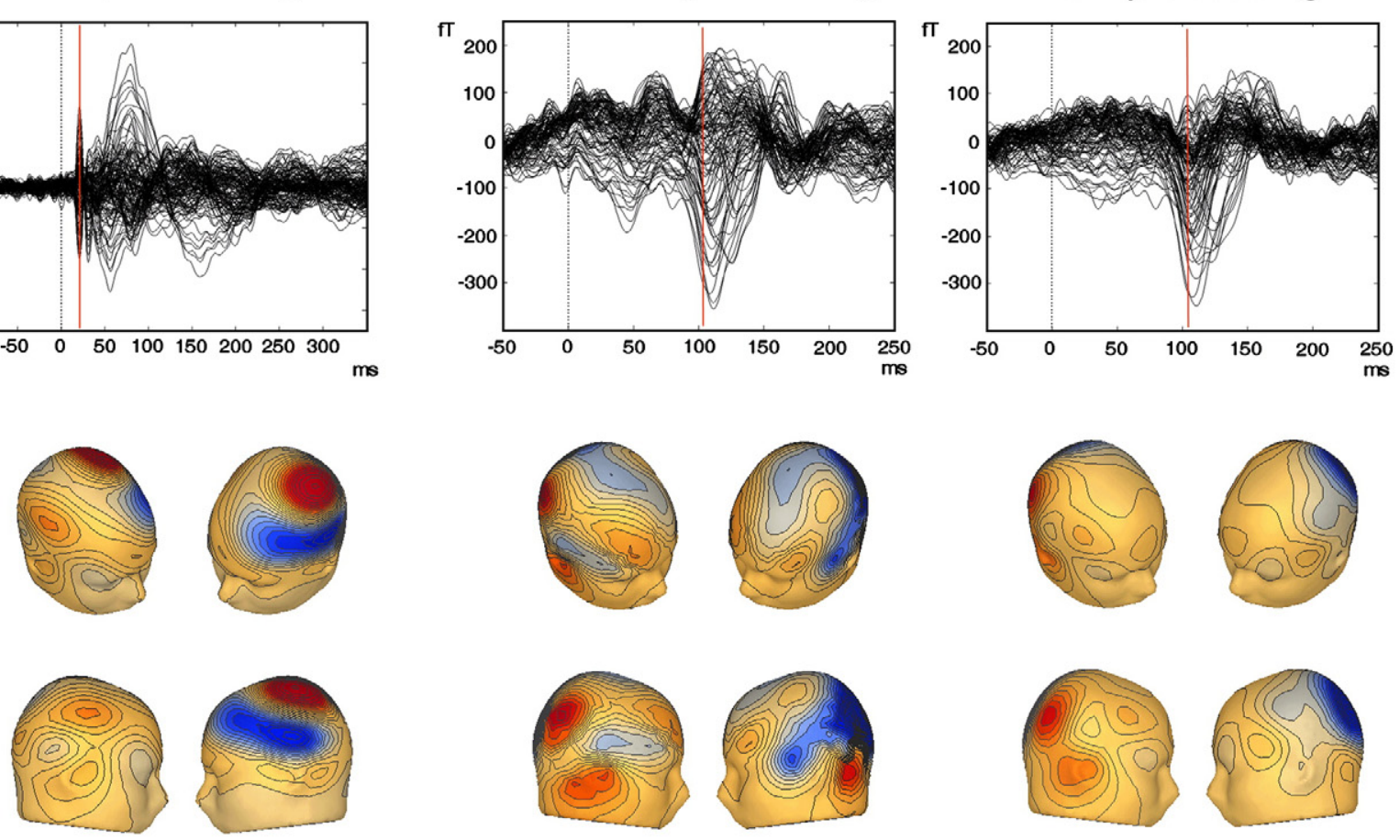

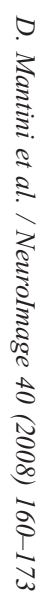

$21 \mathrm{~ms}$

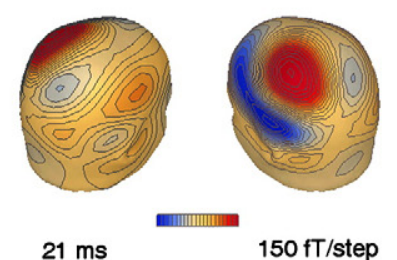

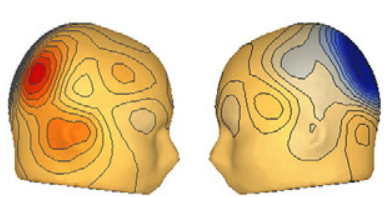

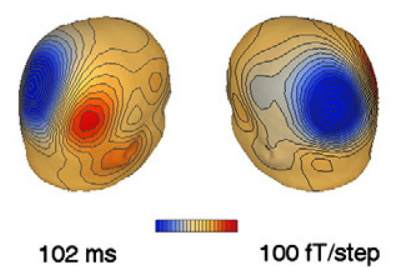

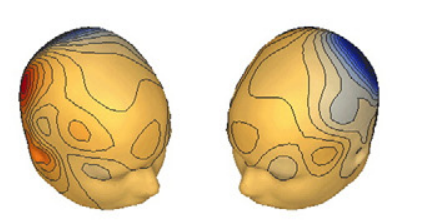

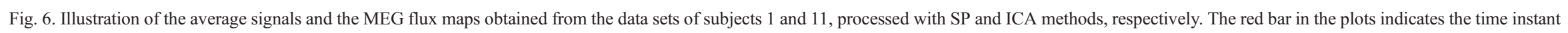
for which the MEG flux maps are shown. 


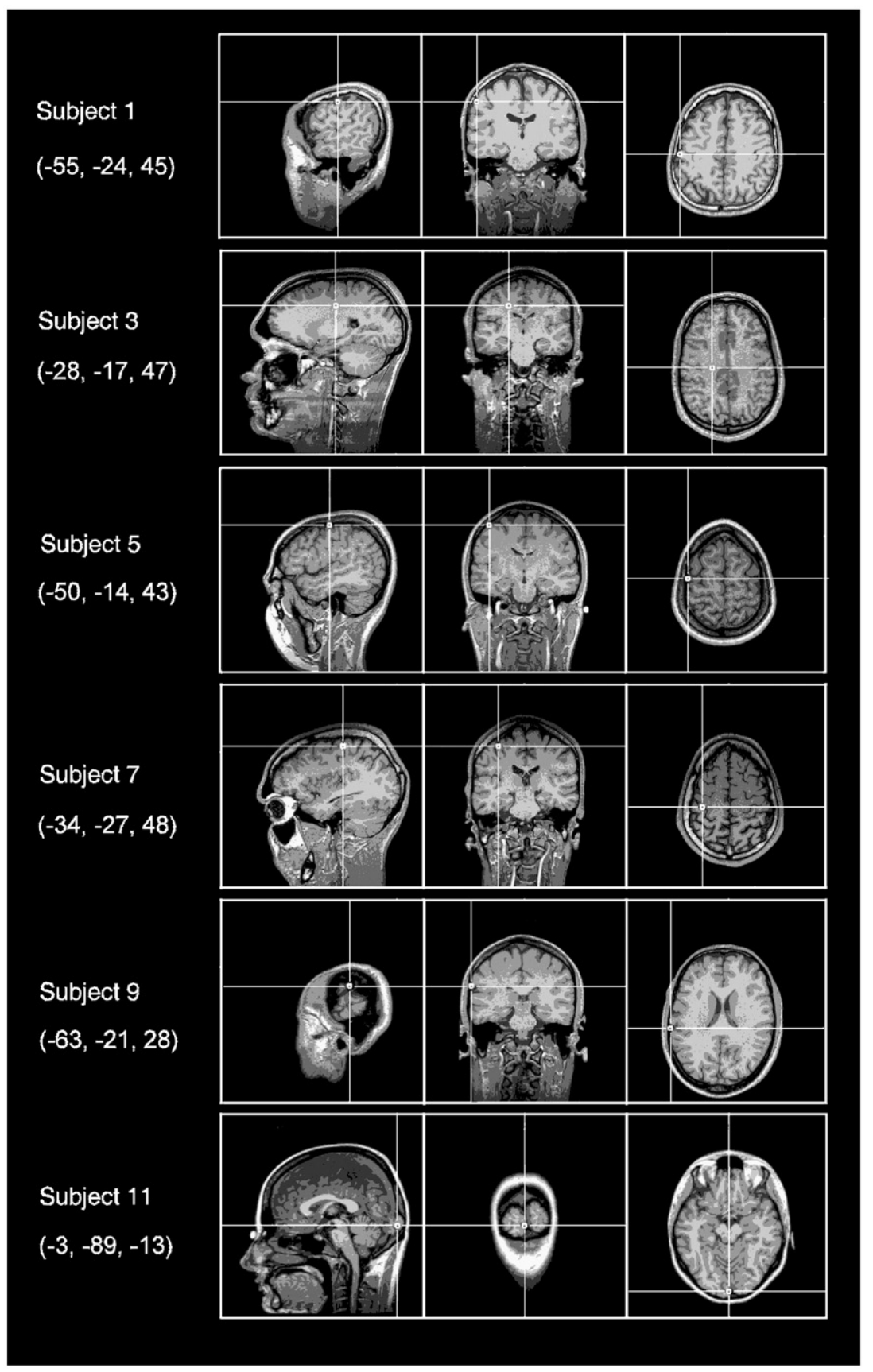

Fig. 7. Dipole localizations for six MEG average signals obtained after ICA, superimposed over the corresponding anatomical images. For each of them, the ECD position in the Talairach coordinates is indicated.

that can be very useful for neuroimaging research (Hari et al., 1997; Pfurtscheller and Lopes da Silva, 1999). However, the compulsory pre-requisite for a relevant use of MEG is the successful separation of high-quality signals, from which it is possible to obtain consistent isofield maps for the solution of the inverse problem (Liljestrom et al., 2005; Phillips et al., 1997). The task of reconstructing reliable and clear MEG traces is not easy, because cerebral activity is generally masked by noise from biological or technical origins. As a consequence, the availability of a standardized procedure for the elimination of such disturbances would be extremely useful for the study of the human brain function. In this work, we have presented an automated method based on ICA for the retrieval of high-quality
MEG signals. Since ICA can be considered an advanced noise reduction method, which not only separates noise sources from the brain-related signals but also separates brain signals from each other, it is our opinion that the development of proposed system might represent a further step toward the study of signals associated with event-related activity and ongoing activity in the human brain.

\section{Acknowledgments}

The authors are grateful to Srinivasan Vairavan for the ApEn software code, to Francesca Petrucci for methodological support, 
Table 4

Localization performances using the processed MEG data

\begin{tabular}{|c|c|c|c|c|c|c|c|}
\hline & \multirow{2}{*}{$\begin{array}{l}\text { Localization time } \\
\text { interval }\end{array}$} & Subject 1 & Subject 3 & Subject 5 & Subject 7 & Subject 9 & Subject 11 \\
\hline & & $18-23$ & $32-42$ & $25-31$ & $54-60$ & $150-170$ & $100-105$ \\
\hline \multirow[t]{3}{*}{$\mathrm{SP}$} & Signal-to-noise ratio & 10.1 & 4.1 & 5.4 & 8.4 & 5.2 & 8.7 \\
\hline & ECD position & $-54,-24,45$ & - & $-56,-20,60$ & $-29,-26,45$ & - & $-12,-70,-19$ \\
\hline & Localization variability & 27 & - & 28 & 38 & - & 43 \\
\hline \multirow[t]{3}{*}{ ICA } & Signal-to-noise ratio & 11.8 & 5.8 & 11.4 & 10.9 & 5.7 & 13.8 \\
\hline & ECD position & $-55,-24,45$ & $-28,-17,47$ & $-50,-14,53$ & $-34,-27,48$ & $-63,-21,28$ & $-3,-89,-13$ \\
\hline & Localization variability & 18 & 31 & 18 & 24 & 38 & 27 \\
\hline
\end{tabular}

For each subject, the average signals after SP and ICA are used for the ECD localization.

The time interval corresponding to the selected brain wave is specified. The related signal-to-noise ratio, the ECD position in Talairach coordinates and the localization variability are shown. The time interval is expressed in milliseconds after the stimulus onset, whereas position and localization variability are expressed in millimeters.

and to Stefania Della Penna, Maria Stavrinou and Francesco Cianflone for MEG data acquisition.

Availability: A free version of the software for MEG artifact removal based on ICA, implemented in MATLAB, is available at http://www.unich.it/itab/signal.

\section{References}

Barbati, G., Porcaro, C., Zappasodi, F., Rossini, P.M., Tecchio, F., 2004. Optimization of an independent component analysis approach for artifact identification and removal in magnetoencephalographic signals. Clin. Neurophysiol. 115, 1220-1232.

Barbati, G., Sigismondi, R., Zappasodi, F., Porcaro, C., Graziadio, S., Valente, G., Balsi, M., Rossigni, P.M., Tecchio, F., 2006. Functional source separation from magnetoencephalographic signals. Hum. Brain Mapp. 27, 925-934.

Cohen, D., 1972. Magnetoencephalography: detection of the brain's electrical activity with a superconducting magnetometer. Science 175 , 664-666.

Cohen, D., 1968. Magnetoencephalography: evidence of magnetic fields produced by alpha rhythm currents. Science $161,784-786$.

Comon, P., 1994. Independent component analysis - a new concept? Signal Process. 36, 287-314.

Del Gratta, C., Pizzella, V., Tecchio, F., Romani, G.L., 2001. Magnetoencephalography - a non invasive brain imaging method with $1 \mathrm{~ms}$ time resolution. Rep. Prog. Phys. 64, 1759-1814.

Del Gratta, C., Della Penna, S., Ferretti, A., Franciotti, R., Pizzella, V., Tartaro, A., Torquati, K., Bonomo, L., Romani, G.L., Rossini, P.M., 2002. Topographic organization of the human primary and secondary somatosensory cortices: comparison of fMRI and MEG findings. NeuroImage 17, 1373-1383.

Della Penna, S., Del Gratta, C., Granata, C., Pasquarelli, A., Pizzella, V., Rossi, R., Russo, M., Torquati, K., Erne, S.N., 2000. Biomagnetic systems for clinical use. Philos. Mag. 80, 937-948.

Della Penna, S., Brancucci, A., Babiloni, C., Franciotti, R., Pizzella, V., Rossi, D., Torquati, K., Rossini, P.M., Romani, G.L., 2007. Lateralization of dichotic speech stimuli is based on specific auditory pathway interactions: neuromagnetic evidence. Cereb. Cortex 17, $2303-2311$.

Fuchs, M., Wagner, M., Kastner, J., 2004. Confidence limits of dipole source reconstruction results. Clin. Neurophysiol. 115, 1442-1451.

Hari, R., Salmelin, R., Makela, J.P., Salenius, S., Helle, M., 1997. Magnetoencephalographic cortical rhythms. Int. J. Psychophysiol. 26, $51-62$.

Hyvärinen, A., 1999. Fast and robust fixed-point algorithms for independent component analysis. IEEE T Neural Netw. 10, 626-634.

Hyvärinen, A., Oja, E., 1997. A fast fixed point algorithm for independent component analysis. Neural Comput. 9, 283-292.
Hyvärinen, A., Oja, E., 2000. Independent component analysis: algorithms and applications. Neural Netw. 13, 411-430.

Jaklevic, R.C., Lambe, J.J., Silver, A.H., Mercereau, J.E., 1964. Quantum interference from a static vector potential in a field-free region. Phys. Rev. Lett. 12, 274-275.

James, C.J., Hesse, C.W., 2005. Independent component analysis for biomedical signals. Physiol. Meas. 26, R15-R39.

Liljestrom, M., Kujala, J., Jensen, O., Salmelin, R., 2005. Neuromagnetic localization of rhythmic activity in the human brain: a comparison of three methods. NeuroImage 25, 734-745.

Makeig, S., Bell, A.J., Jung, T.P., Sejnowski, T., 1996. Independent component analysis of electroencephalographic data. In: Touretzky, D.S. Mozer, M.C., Hasselmo, M.E. (Eds.), Advances in Neural Information Processing Systems 8. MIT Press, Cambridge, pp. 145-151.

Moran, J.E., Drake, C.L., Tepley, N., 2004. ICA methods for MEG imaging. Neurol. Clin. Neurophysiol. 2004, 72.

Nolte, G., Curio, G., 1999. The effect of artifact rejection by signal-space projection on source localization accuracy in MEG measurements. IEEE Trans. Biomed. Eng. 46, 400-408.

Perfetti, B., Franciotti, R., Della Penna, S., Ferretti, A., Caulo, M., Romani, G.L., Onofrj, M., 2007. Low and high frequency evoked responses following pattern reversal stimuli: a MEG study supported by fMRI constraint. NeuroImage 35, 1152-1167.

Pizzella, V., Della Penna, S., Del Gratta, C., Romani, G.L., 2001. SQUID systems for biomagnetic imaging. Supercond. Sci. Technol. 14, R79-R114.

Pfurtscheller, G., Lopes da Silva, F.H., 1999. Event-related EEG/MEG synchronization and desynchronization: basic principles. Clin. Neurophysiol. 110, 1842-1857.

Phillips, J.W., Leahy, R.M., Mosher, J.C., 1997. MEG-based imaging of focal neuronal current sources. IEEE Trans. Med. Imag. 16, $338-348$.

Pincus, S.M., 1991. Approximate entropy as a measure of system complexity. Proc. Natl. Acad. Sci. U. S. A. 88, 2297-2301.

Pincus, S.M., Goldberger, A.L., 1994. Physiological time series analysis: what does regularity quantify? Am. J. Physiol. 266, H1643-H1656.

Rong, F., Contreras-Vidal, J., 2006. Magnetoencephalographic artifact identification and automatic removal based on independent component analysis and categorization approaches. J. Neurosci. Methods 157, $337-354$.

Samonas, M., Petrou, M., Ioannides, A.A., 1997. Identification and elimination of cardiac contribution in single-trial magnetoencephalographic signals. IEEE Trans. Biomed. Eng. 44, 386-393.

Scherg, M., Berg, P., 1991. Use of prior knowledge in brain electromagnetic source analysis. Brain Topogr. 4, 143-150.

Stavrinou, M.L., Della Penna, S., Pizzella, V., Torquati, K., Cianflone, F., Franciotti, R., Bezerianos, A., Romani, G.L., Rossini, P.M., 2007. Temporal dynamics of plastic changes in human primary somatosensory cortex after finger webbing. Cereb. Cortex 17, 2134-2142. 
Taulu, S., Kajola, M., Simola, J., 2004. Suppression of interference and artifacts by the Signal Space Separation Method. Brain Topogr. 16, 269-275.

Uusitalo, M.A., Ilmoniemi, R.J., 1997. Signal-space projection method for separating MEG or EEG into components. Med. Biol. Eng. Comput. 35, 135-140.

Vigário, R., Jousmäki, V., Hämäläinen, M., Hari, R., Oja, E., 1998. Independent component analysis for identification of artifacts in magnetoencephalographic recordings. In: Jordan, M.I, Kearns, M.J., Solla, S.A. (Eds.), Advances in Neural Information Processing Systems, vol. 10. MIT Press, Cambridge, pp. 229-235.
Vrba, J., Robinson, S.E., 2001. Signal processing in magnetoencephalography. Methods 25, 249-271.

Wagner, M., Fuchs, M., Kastner, J., 2004. Evaluation of sLORETA in the presence of noise and multiple sources. Brain Topogr. 16, 277-280.

Williamson, S.J., Lu, Z.L., Karron, D., Kaufman, L., 1991. Advantages and limitations of magnetic source imaging. Brain Topogr. 4, 169-180.

Zhou, X.H., Obuchowski, N.A, McClish, D.K., 2002. Statistical Methods in Diagnostic Medicine. Wiley-Interscience, New York.

Ziehe, A., Nolte, G., Sander, T., Muller, K.R., Curio, G., 2000. Artifact reduction in magnetoneurography based on time-delayed second-order correlations. IEEE Trans. Biomed. Eng. 47, 75-87. 